\title{
Synthesis of Pyromellitic Dianhydride (PMDA) and Tetraoctyl Pyromelli- tate (TOPM) by Alkali Oxygen Oxidation of Anthraeite and Esterification of PMDA with Isooctyl Alcohol
}

\author{
Kun $\mathrm{Du}^{*}$, Yu Li, Zhifang Fan \& Hong Wu
}

Department of Chemistry and Chemical Engineering, Liu Pan Shui Normal University, Liupanshui, Guizhou, China

kundu408@163.com

Keyword: Pyromellitic dianhydride; Tetraoctyl Pyromellitate; selectively decarboxylation; esterification

\begin{abstract}
Pyromellitic dianhydride (PMDA) was prepared by an alkali oxygen oxidation of anthracite and selectively decarboxylation of pyromellitic acid (PA). Tetraoctyl Pyromellitate (TOPM) was synthesized by the esterification of PMDA with isooctyl alcohol. The influence of temperature and time on the yield of PMDA was discussed. The effect of the amount of catalyst, weight ratio of PMDA and isooctyl alcohol on the percent conversion of TOPM was also investigated. The results indicate that worthy chemicals such as PMDA and TOPM can be prepared from coal.
\end{abstract}

\section{INTRODUCTION}

It is very important to develop effective methods for utilizing anthracite, which are most abundant fossil resources. Benzene polycarboxylic acid (BPCA) and its ramification are important materials of organic chemistry, and they are basic materials for the high value-added product [Song C,2002][Liu Fang-Jing, 2013][Wang Wenhua, 2013]. They usually produced from oxidation of aromatic hydrocarbon under the condition of high temperature, high pressure, etc. The drawback of the method is the high production cost, inactivation of catalyst and serious environment pollution, etc. To overcome the drawback, several methods have been proposed, and preparation of BPCA from coal is one of such methods [Calemma V,1994][Miurak, 2000][Miura, 2001].

Many aromatic and heteroatom containing compounds can be converted into monomers for high performance polymers, and many of these monomers can be obtained by oxidation of corresponding alkyl aromatics.

Polish Academy of Sciences Institute of Organic Chemistry studied decarboxylation dynamics using benzene-pentacarboxylic acid and benzene-hexacarboxylic acid as materials in the early 1970's. The reaction is under room temperature, and in the mixed medium of concentrated $\mathrm{H}_{2} \mathrm{SO}_{4}$ and $\mathrm{KHSO}_{4}$. Benzene-pentacarboxylic acid and benzene-hexacarboxylic acid firstly dehydrated to form symmetrical dianhydride under $300{ }^{\circ} \mathrm{C}$, and then converted into pyromellitic dianhydride through selectively decarboxylation when the temperature higher than $300{ }^{\circ} \mathrm{C}$. In 1986 , Salbut et al. investigated pyromellitic acid production from coal in the two-stage process of oxidation and decarboxylation. Chemical ways of aromatic polycaraboxylic acids conversion to such valuable byproducts as phthalic, isophthalic or pyromellitic acids (PA) are presented [Salbust P D, 1986][Schlenk H, 1960]. In 1994, Dalian university of technology explored different coal type's Efficiency of controlled coal oxidation in an alkaline medium for the aim of preparing PA. The results indicate that $7 \sim 8 \%$ (daf) pyromellitic acid can be obtained from Yangquan anthracite.

The aim of this study was to through the two steps: alkali oxygen-oxidation and selectively decarboxylation for preparing PA. After that, used PA, which prepared from coal, and isooctyl alcohol to prepare tetraoctyl Pyromellitate (TOPM), a kind of plasticizer. Extend the downstream of coal and achieve the aim of coal's clean utilize. The structure of Pyromellitic dianhydride (PMDA) and Tetraoctyl pyromellitate (TOPM) were characterized by Fourier transform spectrometer (FT-IR), 
$1 \mathrm{H}$ nuclear magnetic resonance spectra $\left({ }^{1} \mathrm{H}-\mathrm{NMR}\right)$ and $13 \mathrm{C}$ nuclear magnetic resonance spectra $\left({ }^{13} \mathrm{C}-\mathrm{NMR}\right)$.

\section{EXPERTMENTAL SECTION}

\subsection{Materials}

Jingmei anthracite, which purchased from Yunnan was grounded to less than $0.35 \mathrm{~mm}$ particles then dried for $24 \mathrm{~h}$ before used. The detailed analysis of Jingmei anthracite is shown in Table 1 and Table 2, respectively.

Table 1. Proximate analyses (wt \%) of Jingmei anthracite

\begin{tabular}{cccc}
\hline $\mathrm{M}_{\mathrm{ad}}$ & $\mathrm{A}_{\mathrm{d}}$ & $\mathrm{V}_{\mathrm{daf}}$ & $\mathrm{FC}_{\mathrm{ad}}$ \\
\hline 2.983 & 19.88 & 4.831 & 72.29 \\
\hline
\end{tabular}

Table 2. Ultimate analyses (wt \%) of Jingmei anthracite

\begin{tabular}{lcccc}
\hline $\mathrm{C}$ & $\mathrm{H}$ & $\mathrm{N}$ & $\mathrm{O}_{\text {diff }}$ & $\mathrm{S}_{\text {t.d }}$ \\
\hline 69.32 & 2.94 & 0.92 & 26.82 & 1.99 \\
\hline
\end{tabular}

Concentrated $\mathrm{H}_{2} \mathrm{SO}_{4}(98 \%), \mathrm{KHSO}_{4}$, dilute hydrochloric acid, potassium hydroxide, isooctyl alcohol, tetrabutyl titanate, active carbon, activated clay, butanone and 2-pentanone are all purchased from Alpha Chemical Co.,Ltd. Zhengzhou., and were used as received without further purification.

\subsection{Alkali-oxygen oxidation of coal in aqueous solution of potassium hydroxide}

A $50 \mathrm{~g}$ of Jingmei anthracite, $50 \mathrm{~g}$ of potassium hydroxide, and $160 \mathrm{~g}$ of distilled water were put into a $1.5 \mathrm{~L}$ autoclave. The autoclave was heated to $280{ }^{\circ} \mathrm{C}$ and a pressure of $6.0 \mathrm{MPa} \mathrm{O}_{2}$ (cold) was used. The reaction time was $1.0 \mathrm{~h}$ while the reactor was agitated at an average of $500 \mathrm{cycles} / \mathrm{min}$ to keep reactants well mixed. The reaction products were filtered with distilled water to remove insoluble residue (cinder + mineral) after the reaction was over.

The $\mathrm{pH}$ of the filtrate was acidized by Concentrated $\mathrm{H}_{2} \mathrm{SO}_{4}(98 \%)$ to 3.8 and the humic acid was filtrated and throw away. The $\mathrm{pH}$ of the filtrate was acidized by Concentrated $\mathrm{H}_{2} \mathrm{SO}_{4}(98 \%)$ once again to 1.7, and the produced humic acid was also filtrated and throw away again. After that, the filtrate was extracted by butanone time and again, and the butanone was recoveried by a vacuum rotary drier. The remaining concentration was dried to get BPCA.

\subsection{Selectively decarboxylation of $B P C A$ and the refining of PMDA}

$50 \mathrm{~g}$ BPCA, $150 \mathrm{~g}$ concentrated $\mathrm{H}_{2} \mathrm{SO}_{4}, 350 \mathrm{~g} \mathrm{KHSO}_{4}$ (the weight ratio of BPCA, concentrated $\mathrm{H}_{2} \mathrm{SO}_{4}$ and $\mathrm{KHSO}_{4}$ were 1:3:7) and $400 \mathrm{ml}$ distilled water were charged into a $1000 \mathrm{ml}$ threenecked flask while adding a little zeolite to the flask. The three-necked flask was equipped with an air set pipe and was put in a sand bath furnace, and heated the sand bath furnace to $300{ }^{\circ} \mathrm{C}$ and maintained for $2 \mathrm{~h}$.

After that, cooling the reaction products slightly and filtered them, and then the filtrate was extracted by butanone for five times. The exctraction was concentrated under vacuum rotary evaporation. The butanone was recovered, and the extraction was dried in a vacuum drying oven to get crude PA.

Adding 2-pentanone to crude pyromellitic acid under constant stirring, heated the solution to the boiling point of 2-pentanone. Then adding activated charcoal and the hot solution was filtration to remove insoluble impurities; the filtrate was cooled, and the pyromellitic acid crystal was precipitated. Using 2-pentanone washed the crystals several times and the crystals were heated in vacuum chamber at $260^{\circ} \mathrm{C}$ for $3 \mathrm{~h}$ to get pure PMDA. 
Fixing the reaction time at $2 \mathrm{~h}$ and performed the selectively decarboxylation of BPCA at $250{ }^{\circ} \mathrm{C}, 300{ }^{\circ} \mathrm{C}, 350{ }^{\circ} \mathrm{C}$ and $400{ }^{\circ} \mathrm{C}$, respectively to study the influence of the reaction time to the yield of PA.

Fixing the reaction temperature at $350{ }^{\circ} \mathrm{C}$ and performed the selectively decarboxylation of BPCA at $15 \mathrm{~min}, 30 \mathrm{~min}, 45 \mathrm{~min}$ and $60 \mathrm{~min}$, respectively to study the influence of the reaction temperature to the yield of PA.

\subsection{Synthesis of TOPM}

1 mole pure PMDA, which prepared from coal, 5 mole isooctyl alcohol and $5 \mathrm{~g}$ tetrabutyl titanate were put into a $1000 \mathrm{ml}$ three-necked flask equipped with thermometer, water knockout drum and reflux condensing tube. The mixture was heated from atmospheric temperature to $220{ }^{\circ} \mathrm{C}$ and controled the reaction temperature to $220 \pm 10{ }^{\circ} \mathrm{C}$. The generated water was periodically measured from the water knockout drum, and stopped the reaction when the acid number of the product under $0.2 \mathrm{mgKOH} / \mathrm{g}$.

The three-necked flask was linking-up to a vacuum pump to pull off the remaining isooctyl alcohol until there was no isooctyl alcohol can be seen in the reflux condensing tube. And then, $1 \%$ active carbon of the products was added to decolorize. Afterwards, the products which through decolorize were put into a sand core funnel to carry out vacuum suction filter, and $10 \mathrm{~cm}$ activated clay was spread out in the sand core funnel as filter aid.

Fixing the reaction temperature at $220^{\circ} \mathrm{C}$, changed the weight ratio of PMDA and isooctyl alcohol at 1:1, 1:3, 1:5 and 1:7, respectively to investigate the effect of weight ratio of PMDA and isooctyl alcohol on the percent conversion of PMDA.

Fixing the reaction temperature at $220{ }^{\circ} \mathrm{C}$ and the weight ratio of PMDA and isooctyl alcohol at $1: 5$, changed the amount of tetrabutyl titanate catalyst at $1 \%, 3 \%, 5 \%$ and $8 \%$ (basied on the total weight of PMDA and isooctyl alcohol) to investigate the effect of weight ratio of catalyst on the percent conversion of PMDA.

\section{RESULTS AND DISCUSSION}

\subsection{Influence of temperature on the yield of $P A$}

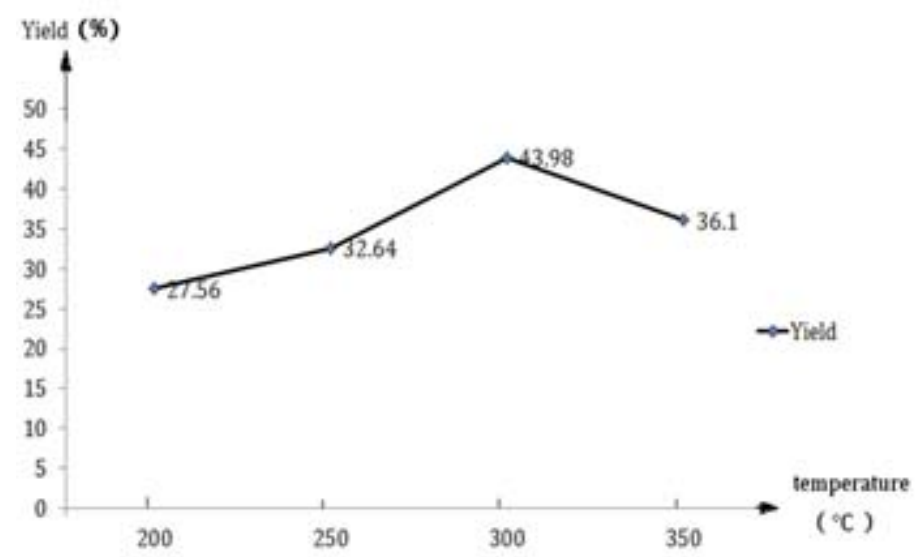

Figure 1. Effect of temperature on the PA yield.

Figure 1 gives the influence of temperature on the crude PA yield. When the time of the selectively decarboxylation of BPCA keep at $2 \mathrm{~h}$, the yield of PA continue to show an upward tendency directly with temperature while the time of the selectively decarboxylation of BPCA keep at $2 \mathrm{~h}$. The yield of PA reached its highest value: $43.98 \%$. The yield of PA decreased when the temperature continue to rise. 
The results illustrate that temperature has great influence on the yield of PA. The reaction speed is very slow and it needs a long time to selectively decarboxylate, and the yield of PA is quite low. Obviously, it doesn't really amount to much. But then, too high temperature will makes PA further decomposed, and it also lead to the yield of PA decrease. The most suitable temperature for selectively decarboxylation is $2 \mathrm{~h}$.

\subsection{Influence of reaction time on the yield of $P A$}

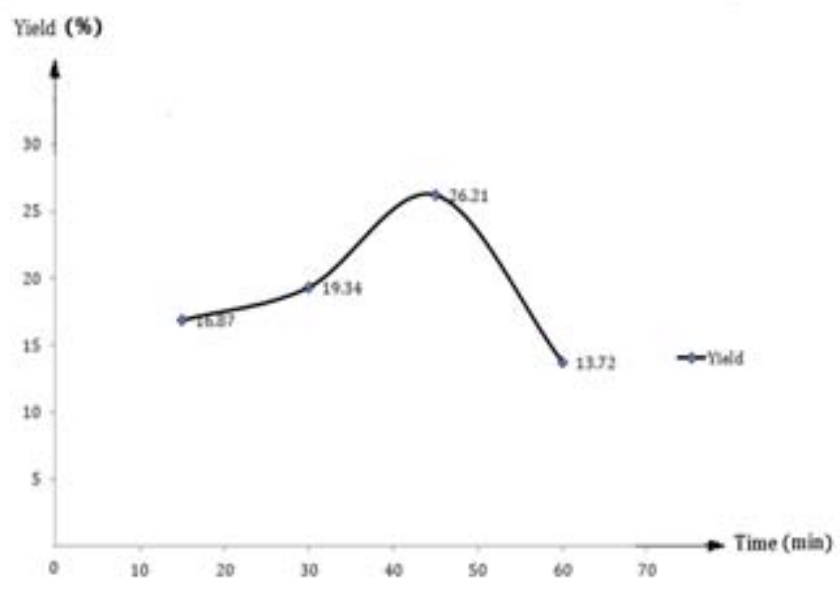

Figure 2. Effect of reaction time on the PA yield.

Figure 2 shows the effect of reaction time on the PA yield. The selectively decarboxylation reaction of BPCA is insufficient when the reaction time is relatively short, and the yield of PA is rather low. It presents the tendency of increase with the extension of the reaction time. The yield of PA begins to decrease after it reaches its peak value.

It is generally supposes that the process of coal oxidation is: Coal $\rightarrow$ humic acid $\rightarrow$ coal acids $\rightarrow$ $\mathrm{CO}_{2}+\mathrm{H}_{2} \mathrm{O}$. The first stage engages in the surface of coal particles, and its speed is comparatively rapid. The second stage engages in the liquid phase, and its speed is slower than the first stage. Too long reaction time makes the speed of the third stage accelerate, then the PA is be further oxidized. The result is that the ultimate yield of PA decreases, and the results illustrate that 45 minutes is the most favourable time.

\subsection{Influence of weight ratio of PMDA and isooctyl on the percent conversion of PMDA}

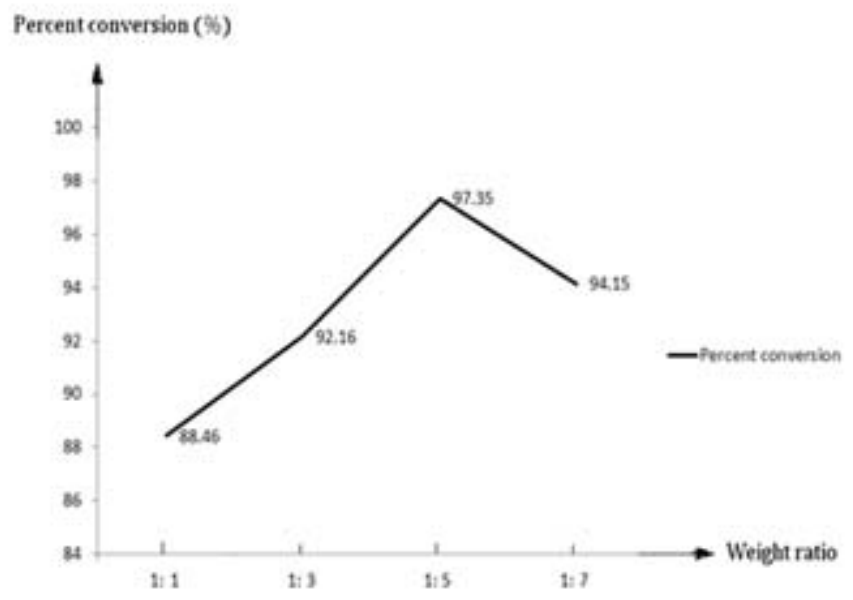

Figure 3. Effect of weight ratio of PMDA and isooctyl on the percent conversion of PMDA.

The weight ratio of PMDA and isooctyl has great influence on the percent conversion of PMDA by looking at Figure 3. The higher of the weight ratio of PMDA and isooctyl leads to the higher of 
the percent conversion of PMDA. That is to say, higher weight ratio of PMDA and isooctyl is beneficial for the percent conversion of PMDA. But too high weight ratio of PMDA and isooctyl has rendered the pressure of the reaction rise, and it involves the difficulty of isooctyl reflux. It turns the percent conversion of PMDA decrease.

\subsection{Influence of weight ratio of catalyst on the percent conversion of PMDA}

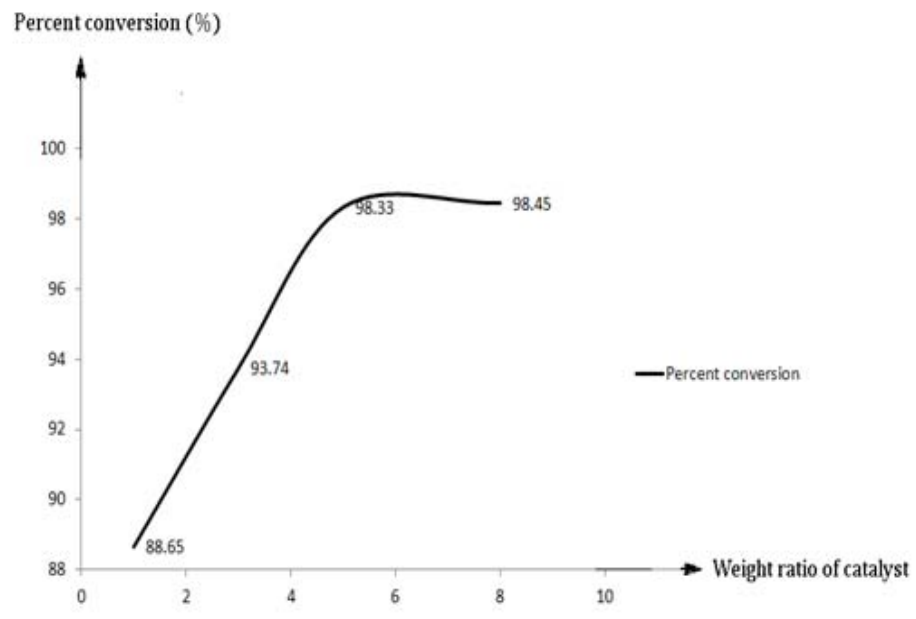

Figure 4. Effect of weight ratio of catalyst on the percent conversion of PMDA.

We can recognized from Figure 4 that the more quantity of catalyst adding involve higher percent conversion of PMDA. But the percent conversion of PMDA has not obviously increased when the adding of catalyst exceeded 5\%. Besides, more catalyst adding increases the difficulty of production post-treating.

\subsection{Analysis of PMDA and TOPM}

The structure of PMDA was confirmed by ${ }^{1} \mathrm{H}-\mathrm{NMR}$ and ${ }^{13} \mathrm{C}-\mathrm{NMR}$, and the structure of TOPM was confirmed by ${ }^{1} \mathrm{H}-\mathrm{NMR}$ and FT-IR.

FT-IR spectral data were obtained on a Digilab FTS-20E system by using $\mathrm{KBr}$ disc techniques. The mixture of 0.8 to $1.2 \mathrm{mg}(+0.01 \mathrm{mg})$ of sample and $95-105 \mathrm{mg} \quad(+0.01 \mathrm{mg})$ of $\mathrm{KBr}$ was finely ground for at least 15 minutes to assure the optimum measurement and then pressed under vacuum with a load of $8000 \mathrm{~kg}$.

The ${ }^{1} \mathrm{H}-\mathrm{NMR}$ spectra were recorded in deuteriochloroform solutions in the usual way. As for ${ }^{13} \mathrm{C}$-NMR, the sample were recorded in $\left(\mathrm{CD}_{3}\right)_{2} \mathrm{SO}$ solutions, and a relaxation agent, chromium trisacetylacetonate $(0.1 \mathrm{M})$ was added in an inverse gated decoupling system to get quantitative ${ }^{13} \mathrm{C}$ NMR spectroscopy.

As for the ${ }^{1} \mathrm{H}-\mathrm{NMR}$ of PMDA, there is only one kind of proton: $8.0(\mathrm{Ar}-\mathrm{H})$ appears at 8.73 ppm. The sharp peak at $2.5 \mathrm{ppm}$ could be assigned to $\left(\mathrm{CD}_{3}\right)_{2} \mathrm{SO}$ solvent peak and the small impurity peak appears at $8.27 \mathrm{ppm}$.

For the ${ }^{13} \mathrm{C}$-NMR of PMDA, the carbonyl carbon atom exhibits at $161.9 \mathrm{ppm}$; the tertiary carbon atom shows at $137.6 \mathrm{ppm}$ and the quaternary carbon atom appears at $124.8 \mathrm{ppm}$. The heights of the spectrum are broadly in line with $2: 2: 1$, which reflected the type and number of carbon atoms in PMDA. Some petty impurity peaks display at 121.7, 133.1, 139.4, 161.3 and $166.8 \mathrm{ppm}$.

As for the FT-IR of TMOP, The $-\mathrm{CH}_{3}$ - and $-\mathrm{CH}_{2}$ - absorption show intense peak at 2800 to $3000 \mathrm{~cm}^{-1}$, and the characteristic bands of $1730 \mathrm{~cm}^{-1}$ is the $(-\mathrm{C}=\mathrm{O})$ stretching vibration. The vibration of asymmetric bending is shown at $1459 \mathrm{~cm}^{-1}$ and the strong sharp peak at $1104 \mathrm{~cm}^{-1}$ is attributed to $\left(-\mathrm{OCH}_{2} \mathrm{CH}_{2}-\right)$ stretching vibration. The $(-\mathrm{C}=\mathrm{C}-)$ stretching is shown a sharp intense peak at $1643 \mathrm{~cm}^{-1}$. The $\mathrm{C}=\mathrm{C}$ stretching is shown as a sharp intense peak at $1622 \mathrm{~cm}^{-1}, 1023 \mathrm{~cm}^{-1}$ and 952 $\mathrm{cm}^{-1}$ for $\mathrm{C}-\mathrm{H}$ plane deformation vibration of $=\mathrm{CH}_{2}$. This 1602 and $1502 \mathrm{~cm}^{-1}$ absorptions are at- 
tributed to the existence of benzene nucleus. The FTIR proves that the synthesized monomer has the anticipated structure.

For the ${ }^{1} \mathrm{H}-\mathrm{NMR}$ of TOPM, the signal at $\delta=8.6 \mathrm{ppm}$ assigned to the $(\mathrm{Ar}-2 \mathrm{H})$. The chemical shifts at $\delta=4.28 \mathrm{ppm}$ and $\delta=4.21 \mathrm{ppm}$ resulting from the ether $\left(\mathrm{CH}_{2}-\mathrm{O}-\mathrm{CH}_{2}-, 8 \mathrm{H}\right)$ groups. $\delta=1.51$, $1.34,1.00,0.92,0.88 \mathrm{ppm}$ were the high coupling peak at the structure of $-\mathrm{CH}-\mathrm{CH}_{2}-\mathrm{CH}_{2}-\mathrm{CH}_{2}-\mathrm{CH}_{3}$. From FT-IR measurements and ${ }^{1} \mathrm{H}-\mathrm{NMR}$ analysis, it can confirm that synthetic TOPM have the expected structure.

\section{CONCLUSION}

1. The authors have prepared pure PMDA and TOPM from anthracite, and survived better reaction conditions for the two compounds. Studied multifarious factors influenced on the yield of the two compounds.

2. Under optimal conditions, the yield of PMDA is $14.7 \%$ based on the weight of BPCA and $11.2 \%$ based on the weight of daf. coal. The yields of TOPM is $11.21 \%$ based on the weight of BPCA and $6.57 \%$ based on the weight of daf. coal.

3. The optimal conditions for PMDA production: $300{ }^{\circ} \mathrm{C}, 45 \mathrm{~min}$ and the PMDA purity can be further improved by recycling the mother liquid of 2-pentanone recrystallization. The optimal conditions for TOPM production: $220{ }^{\circ} \mathrm{C}, 45 \mathrm{~min}$, and the weight ratio of PMDA and isooctyl alcohol is $1: 5$.

\section{ACKNOWLEDGES}

The authors gratefully acknowledge the support of the Solid Waste Recycling Laboratory of Coal Utilization (NO: 2011-278), the Guizhou Provincial Department of Education 2011 Key Research Projects (NO: [2011]232) and Guizhou Provincial Department of S\&T 2011 Projects of Social Development (NO: SY 20113112).

\section{REFERENCES}

[1] Song C et al. 2002. Chemicals and materials from coal in the $21^{\text {st }}$ century. Fuel. 81(1): 15-32.

[2] Liu Fang-Jing et al. 2013. Characterization of biomarkers and structural features of condensed aromatics in Xianfeng lignite. Energy \& Fuels. 27: 7369-7378.

[3] Wang Wenhua et al. 2013. Production of benzene polycarboxylic acids from bituminous coal by alkali-oxygen oxidation at high temperatures. Fuel Processing Technology. 110: 184-189.

[4] Calemma V et al. 1994. Changes in coal structure accompanying the formation of regenerated humic acids during air oxidation. Fuel. 73(5): 700-707.

[5] Miurak. 2000. Mild conversion of coal for producing valuble chemicals. Fuel Processing Tecnology. 62(2-3): 119-135.

[6] Miura Kouichi et al. 2001. A new two-step oxidative degradation method for producing valuable chemicals from low rank coals under mild conditions. Energy \& Fuels. 15: 611-617.

[7] Salbust P D. 1986. Improvements in the process of oxidation of coal to aromatic polycarboxylic acids. Przemysl Chemiczny. 65(1): 23-25.

[8] Schlenk H et al. 1960. Esterification of fatty acids with diazomethane on a small scale. Analytical Chemistry. 32(11): 1412-1414. 